\title{
Diagnóstico de la relación entre el uso excesivo de las TICs y síntomas depresivos y ansiedad en estudiantes de medicina, Universidad de Cuenca, Ecuador
}

\author{
José I. Ortiz S. , Manuel I. Morocho M. ${ }^{1}$, Ángel H. Tenezaca T. ${ }^{1}$, María G. Torres O. ${ }^{2}$, Diana P. \\ Ugalde T. \\ ${ }^{1}$ Profesor titular de la Facultad de Ciencias Médicas, Universidad de Cuenca, Avenida El Paraíso s/n, \\ junto al Hospital Vicente Corral, Cuenca, Ecuador. \\ ${ }^{2}$ Estudiante de la Carrera de Medicina, Facultad de Ciencias Médicas de la Universidad de Cuenca, \\ Ecuador.
}

Autor para correspondencia: jose.ortiz@ucuenca.edu.ec

Fecha de recepción: 2 de agosto de 2014 - Fecha de aceptación: 16 de septiembre de 2014

\begin{abstract}
RESUMEN
Se trata de un estudio descriptivo de corte transversal entre estudiantes de la Facultad de Ciencias Médicas de la Universidad de Cuenca ${ }^{1}$, para determinar la magnitud del uso excesivo de Tecnologías de Información y Comunicación (TICs), su variación en función de la edad, sexo, estado civil y la carrera; así como su asociación con síntomas depresivos y de ansiedad. En el cuestionario previamente validado que fue aplicado a muestra aleatoria por conglomerados de 333 estudiantes, se integraron preguntas de información demográfica, test de uso excesivo de TICs y de síntomas depresivos y de ansiedad. El concepto de Riesgo Relativo y su Intervalo de Confianza al 95\% fue utilizado para el análisis estadístico. Los resultados nos revelan que la edad promedio de la muestra de la población fue de 22 años, 37,2\% con uso excesivo de TICs, el 18,3\% presentan síntomas depresivos y 39,8\% de ansiedad. Entre las variables asociadas de manera significativa con el uso excesivo de TICs están: la edad menor de 19 años (RR 2,04; IC 95\% 1,31-3,20), el género masculino (RR 1,36; IC 95\% 1,031,80 ) y la carrera de Tecnología Médica (RR 1,83; IC 95\% 1,28-2,63). El uso excesivo de las TICs no está asociado con el estado civil. Sin embargo, el análisis estadístico nos muestra la existencia de una correlación positiva entre el uso excesivo de las TICs y la frecuencia de estudiantes con síntomas depresivos (RR 1,88; IC 95\% 1,45-2,44) y de ansiedad (RR 2,61; IC 95\% 1,64-4,15).
\end{abstract}

Palabras clave: Uso excesivo de TICs, síntomas depresivos y de ansiedad.

\begin{abstract}
A descriptive cross-sectional study among students of the Faculty of Medicine of the University of Cuenca was conducted to assess the magnitude of the excessive use of Information and Communication Technology (ICT), the variation in terms of age, sex, marital status and career, and its association with depressive and anxiety symptoms. The validated questionnaire submitted to a random cluster of 333 students, included in addition to the gathering of demographic information, questions probing the use of ICT and depressive and anxiety symptoms. The concept of Relative Risk associated with a Confidence Interval of $95 \%$ was used in the statistical analysis. Results revealed that the average age of the sample population was 22 years, 37,2\% use ICT excessive, and 18,3\% show depressive symptoms and 39,8\% signs of anxiety. Variables significantly associated with the excessive use of ICT are students younger than 19 years (RR 2,04; CI 95\% 1,31-3,20), male gender (RR 1,36; CI

Esta investigación forma parte del proyecto Tecnologías de Información y Comunicación en la formación de los Recursos Humanos en Salud, que fue financiado por el Consorcio Ecuatoriano para el Desarrollo de Internet Avanzado (CEDIA).
\end{abstract}


95\% 1,03-1,80) and the Medical Technology field of study (RR 1,83; CI 95\% 1,28-2,63). Excessive use of ICT is not associated with the marital status. However, the statistical analysis revealed the existence of a significant positive correlation between problematic ICT use and the frequency of students with depressive symptoms (RR 1,88; CI 95\% 1,45-2,44) and anxiety (RR 2,61; CI 95\% 1,64$4,15)$.

Keywords: Excessive use of ICTs, depressive and anxiety symptoms.

\section{INTRODUCCIÓN}

Las Tecnologías de la Información y la Comunicación (TICs) constituyen el conjunto de recursos, procedimientos y técnicas usadas para la búsqueda, elaboración, almacenamiento y transmisión de información, que se obtiene a través del teléfono celular, tableta, computadora y otros aparatos, que se han integrado a nuestro quehacer individual, social y académico, de manera creciente en los últimos años, lo cual está influyendo en los cambios de conducta y en la generación de estados de ánimo algunas veces patológicos, especialmente en quienes utilizan de forma desmedida. Aunque tradicionalmente los términos de adicción y dependencia han sido considerados para referirse a los problemas médicos relacionados con sustancias tóxicas o drogas; así, la adicción como el hábito de quien se deja dominar por el uso de alguna o algunas drogas tóxicas, o por la afición desmedida a ciertos juegos; y la dependencia, como la necesidad compulsiva de alguna sustancia, como alcohol, tabaco o drogas, para experimentar sus efectos o calmar el malestar producido por su privación (Diccionario de la RAE, 2014).

Existen varias investigaciones relacionadas con la adicción al trabajo, Internet, juego, sexo, comida, compras, que sustentan el problema de adicción a las TICs (Echeburúa, 1999; Young y Rogers, 1998; Navarro-Mancilla y Rueda-Jaimes 2007; Castañeda, 2010; Cruzado y col., 2006; Lam y col., 2011; Berner y Santander, 2012). No obstante, en otros estudios se plantea que no existe una adicción a TICs sino problemas de conducta derivados del acceso a la diversidad de contenidos que provee; por lo que el concepto de adicción se debería aplicar cuando el agente nocivo es una sustancia (Yang, 2001; APA, 202; Morrison y Gore, 2010; Domínguez y col., 2012; Fernández, 2013). Al respecto, la comunidad científica encargada de la salud mental ha conceptualizado las adicciones a sustancias de manera exhaustiva y actualmente se presenta el reto de delimitar las adicciones conductuales y sus consecuencias en función de criterios que se relacionan con la necesidad del uso o compulsión, la frecuencia, el tiempo, el dinero invertido, las interferencias con las obligaciones laborales, la vida cotidiana y las relaciones sociales.

En este marco, varios estudios han puesto en evidencia la asociación entre el uso excesivo de las TICs con una gran variedad de trastornos psiquiátricos y dentro de los trastornos de la conducta los síntomas depresivos (Kraut y col., 1998) y de ansiedad. En la investigación realizada por Akin y İskender (2011), en 300 estudiantes universitarios, podemos apreciar una correlación significativa entre la adicción al Internet con la depresión $(r=0,67, p<0,01)$, ansiedad $(r=0,63, p<0,01)$, y el estrés $(\mathrm{r}=0,63, \mathrm{p}<0,01)$. En el estudio de Kyunghee y col. (2005) encontramos que el puntaje de la correlación entre la adicción al Internet con la ideación suicida, fue cuatro veces mayor en adolescentes coreanos adictos al Internet, con respecto a los no adictos $(r=0,45$ versus $r=0,11)$. Según la OMS, la depresión es una enfermedad frecuente en todo el mundo; para hacer el diagnóstico es necesario considerar que el cuadro está presente por lo menos seis meses. Se calcula que afecta a unos 350 millones de personas (OMS, 2012), de aquí la importancia de revisar otras evidencias al respecto.

En un estudio con análisis por conglomerados se encontró que el 4,9\% de la muestra que manifestaban tener problemas frecuentes como sentimientos de culpa, deseo intenso de continuar con la conexión a Internet, pérdida de control, pérdida de tiempo de trabajo o clase, también manifestaban una mayor ansiedad y disfunción social (Blanco y col., 2002). En Girona, España, en una muestra de 1.277 estudiantes universitarios se mostró que el 15\% utilizan diariamente el chat, y de estos el 13,3\% presentan ansiedad (Viñas y col., 2002). En el Reino Unido, el Instituto de Ciencias Psicológicas de la Universidad de Leeds, encontró que el 22,35\% de una muestra de 1.319 jóvenes y adultos, que usan 
Internet en forma excesiva fueron propensos a sufrir de depresión (Morrison y Gore, 2010). Yang (2001) en un estudio en la que participaron 1.296 adolescentes de entre 12 y 19 años, halló que el $8,7 \%$ de los adolescentes que hacían un uso excesivo del ordenador, presentaban ansiedad. En otro estudio realizado con 259 individuos Young y Rogers (1998) describen una asociación entre el uso patológico de Internet con la depresión. En otra investigación también se ha demostrado dicha asociación (Kraut y col., 1998). En Perú, en una muestra de 30 pacientes hospitalizados y con diagnóstico de adicción al Internet se encontró irritabilidad (80\%), merma de rendimiento académico (76,7\%), trastornos afectivos (56,7\%), e intento suicida (2\%) (Cruzado y col., 2006).

Por lo antes mencionado consideramos trascendente estudiar las posibles adicciones conductuales relacionadas con el uso excesivo del teléfono móvil, Internet, juegos y si estas conductas se asocian con algunos trastornos del estado del ánimo que se manifiestan como síntomas depresivos y de ansiedad en los estudiantes de la Facultad de Ciencias Médicas de la Universidad de Cuenca. Sobre la base de los resultados será posible desarrollar actividades y estrategias adecuadas de prevención. El objetivo de esta investigación es determinar la magnitud del uso excesivo de TICs, su variación en función de la edad, sexo, estado civil y la carrera; así como su asociación con la depresión y la ansiedad.

\section{SUJETOS Y MÉTODO}

Realizamos un estudio descriptivo de corte transversal en el que participaron 333 estudiantes de ambos sexos, de entre 17 a 30 años de las carreras de Medicina (178), Enfermería (120), Tecnología Médica (35) de la Universidad de Cuenca, que asistieron regularmente a clases durante el año lectivo entre septiembre de 2012 y julio de 2013, excluyéndose del estudio a aquellos alumnos que reportaron antecedentes de enfermedades mentales o que recibían tratamiento farmacológico para dichos problemas. El cálculo de la muestra se realizó con base en los siguientes parámetros: población total de estudiantes (3359), frecuencia esperada de adicción a TICs 9\%, precisión 3\%, nivel de confianza $95 \%$.

Previo a la recolección de los datos, se consiguió la aprobación por parte de las autoridades y docentes de la Facultad de Ciencias Médicas de la Universidad de Cuenca y el consentimiento informado de los estudiantes. La selección de los individuos se realizó a través del programa que consta en www.randomization.com, utilizando la tercera opción third generator. Se procedió a seleccionar en primer lugar a los cursos de cada carrera y en segundo lugar a los estudiantes de los cursos, teniendo en cuenta la distribución porcentual en cada una de las carreras, a partir de las listas obtenidas en la Secretaría de la Institución. La aplicación de los formularios fue realizado por dos estudiantes de la carrera de medicina previamente entrenadas, durante el período regular de clases en los meses de marzo y abril del año 2012.

Teniendo en cuenta que con la ayuda de los test únicamente podemos detectar síntomas depresivos y de ansiedad, mas no llegar al diagnóstico de depresión y ansiedad, utilizamos un cuestionario con tres grupos de preguntas; en primer lugar, las socio-demográficas elaboradas al efecto y con las que se recogió información acerca de la edad, sexo, estado civil y carrera; en segundo lugar, las que corresponden al uso excesivo de TICs que fueron elaboradas basándonos en los criterios de dependencia a drogas, establecidos por la Asociación Americana de Psiquiatría, que constan en el Manual de Diagnóstico y Estadístico de los Trastornos Mentales DSM-IV-TR ${ }^{2}$ (por sus siglas en inglés) (APA, 2002); y en tercer lugar, las relacionadas con el test de Hamilton (1960), para detectar síntomas depresivos y de ansiedad. El cuestionario fue validado en 30 estudiantes de la Facultad de Odontología, mediante pre-test y re-test, con intervalo de dos semanas, obteniéndose un coeficiente de correlación de 0,76 .

\footnotetext{
2 El Diagnóstico y Estadísticas de las enfermedades Mentales, cuarta edición, DSM-IV-TR (por sus siglas en inglés) es el producto del consenso de la comunidad científica y especialistas relacionados con la salud mental, a nivel mundial, que es escrito en un manual por un comité, en cuyo documento se establecen los criterios para el diagnóstico y tratamiento clínicos, así como estudios estadísticos para la salud pública.
} 
El test de Hamilton (1960) para depresión contiene 18 preguntas graduadas, algunas a 3 (de 0 a 2) y otras a 5 (de 0 a 4) niveles de gravedad; la puntuación total se obtiene sumando las preguntas que puede dar un total de entre 0 a 52 puntos, si el puntaje es igual o mayor de 10, el score es positivo como diagnóstico de depresión y se empieza a considerar indicativa de depresión moderada una puntuación total igual o superior a 18. El test de Hamilton para ansiedad contiene 14 preguntas divididas en ítems para detectar síntomas somáticos musculares y sensoriales, si el puntaje es entre 0 a 5 puntos no existe ansiedad, entre 6 a 14 la ansiedad es menor, y cuando el puntaje es de 15 o más el diagnóstico es de ansiedad mayor. Para este estudio consideramos todos los casos positivos de depresión con puntajes iguales o mayores a 10 y para la ansiedad con puntajes superiores a 5 puntos, sin diferenciar los niveles de gravedad en ambas situaciones.

En el análisis estadístico, para la descripción de las características generales de los participantes utilizamos frecuencias y porcentajes. Para determinar la asociación del uso excesivo de las TICs con los síntomas depresivos y de ansiedad hemos empleado el Riesgo Relativo (RR) y su intervalo de confianza al 95\%; el RR calculamos dividiendo la proporción de estudiantes con síntomas de ansiedad o depresión que están expuestos al uso excesivo de TICs, sobre la proporción de estudiantes con síntomas de ansiedad o depresión que no están expuestos al uso excesivo de TICs. Existen tres posibles resultados, en primer lugar, cuando el RR es menor que uno significa que la probabilidad de presentar síntomas depresivos o de ansiedad es menor en el grupo expuesto a las TICs con respecto a los no expuestos; en segundo lugar, cuando el RR es igual a uno significa que la probabilidad de síntomas depresivos o de ansiedad es el mismo en ambos grupos; y en tercer lugar, cuando el RR es mayor que uno la probabilidad de presentar síntomas depresivos y de ansiedad en el grupo expuesto a las TICs es mayor con respecto a los no expuestos. Para conocer los límites de confianza entre los que se encuentra el verdadero valor del RR utilizamos el intervalo de confianza (IC) al 95\% de acuerdo con la fórmula de Wingo (1996).

\section{RESULTADOS}

En el estudio participaron 333 estudiantes de las carreras de Medicina (53,45\%), Enfermería (36,04\%) y Tecnología Médica (10,51\%), que forman parte de la Facultad de Ciencias Médicas de la Universidad de Cuenca. La mayoría de estudiantes (70,57\%) se ubica en el grupo de entre 19 a 22 años de edad, le sigue el de 23 a 26 (21\%), luego está el grupo de menos de 19 años $(5,41 \%)$, y por último los mayores de 26 años (3\%). El promedio de edad fue de 22 años, con una desviación típica de 2,09. Seis de cada diez participantes fueron mujeres, esto en razón de que en el universo existe una distribución similar. En cuanto al estado civil el $87 \%$ fueron solteros, 7,81\% casados, 3\% unión libre y $0,6 \%$ viudos (ver Tabla 1$)$.

Luego de aplicar el test, encontramos que el 37,2\% de los estudiantes de la Facultad de Ciencias Médicas presenta un uso exagerado de las TICs, lo cual varía en función de algunas características demográficas; así tenemos que, en el grupo menor de 19 años, la probabilidad es algo más del doble (RR 2,04), con respecto a los otros grupos etarios y esta diferencia es estadísticamente significativa (IC 95\% 1,31-3,20); en tanto que las diferencias entre los grupos mayores de 19 años no son significativas. En el sexo masculino existe mayor probabilidad de uso excesivo de TICs $(44,6 \%)$ con respecto al femenino (32,5\%) cuya diferencia es significativa (RR 1,36; IC 95\% 1,03-1,80). La variación entre los distintos grupos de estado civil no es significativa. Al analizar la carrera, podemos ver que los estudiantes de Tecnología Médica presentan un mayor porcentaje de uso excesivo de TICs (62\%), con respecto a Enfermería (30,83\%) y Medicina (36,52\%), esta diferencia es significativa (IC RR 1,83; 95\% 1,28-2,63) frente a los estudiantes de Medicina y Enfermería (Tabla 2). 
Tabla 1. Características demográficas y distribución por carrera de los participantes.

\begin{tabular}{lcc}
\cline { 1 - 2 } Variable & $\mathrm{N}$ & $\%$ \\
\hline$<19$ & 18 & 5,41 \\
$19-22$ & 235 & 70,57 \\
$23-26$ & 70 & 21,02 \\
$>26$ & 10 & 3,00 \\
\hline Sexo & & \\
\hline Femenino & 200 & 60,06 \\
Masculino & 133 & 39,94 \\
\hline Estado civil & & \\
\hline Casado & 26 & 7,81 \\
Divorciado & 5 & 1,50 \\
Soltero & 290 & 87,09 \\
Unión libre & 10 & 3,00 \\
Viudo & 2 & 0,60 \\
\hline Carrera & & \\
\hline Tecnología Médica & 35 & 10,51 \\
Enfermería & 120 & 36,04 \\
Medicina & 178 & 53,45 \\
\hline
\end{tabular}

Tabla 2. Uso excesivo de TICs según edad, sexo, estado civil y carrera.

\begin{tabular}{|c|c|c|c|c|c|c|c|}
\hline \multirow[t]{2}{*}{ Variable } & \multicolumn{2}{|c|}{$\begin{array}{c}\text { Con uso excesivo } \\
\text { de TICs }\end{array}$} & \multicolumn{2}{|c|}{$\begin{array}{c}\text { Sin uso excesivo } \\
\text { de TICs }\end{array}$} & \multirow[t]{2}{*}{ RR } & \multirow{2}{*}{\multicolumn{2}{|c|}{ IC95\% }} \\
\hline & $\mathrm{N}$ & $\%$ & $\mathrm{~N}$ & $\%$ & & & \\
\hline \multicolumn{8}{|l|}{ Edad en años } \\
\hline$<19$ & 13 & 72,22 & 5 & 27,78 & 2,04 & 1,31 & 3,20 \\
\hline $19-22$ & 86 & 36,60 & 149 & 63,40 & 0,94 & 0,69 & 1,27 \\
\hline $23-26$ & 24 & 34,29 & 46 & 65,71 & 0,9 & 0,63 & 1,28 \\
\hline$>26$ & 1 & 10,00 & 9 & 90,00 & 0,26 & 0,06 & 1,12 \\
\hline \multicolumn{8}{|l|}{ Sexo } \\
\hline Masculino & 59 & 44,36 & 74 & 55,64 & 1,36 & 1,03 & 1,80 \\
\hline Femenino & 65 & 32,50 & 135 & 67,50 & & & \\
\hline \multicolumn{8}{|l|}{ Estado civil } \\
\hline Casado & 8 & 30,77 & 18 & 69,23 & 0,81 & 0,46 & 1,43 \\
\hline Divorciado & 1 & 20,00 & 4 & 80,00 & 0,53 & 0,11 & 2,47 \\
\hline Soltero & 111 & 38,28 & 179 & 61,72 & 1,26 & 0,80 & 1,99 \\
\hline Unión libre & 3 & 30,00 & 7 & 70,00 & 0,8 & 0,32 & 1,98 \\
\hline Viudo & 1 & 50,00 & 1 & 50,00 & 1,34 & 0,28 & 6,37 \\
\hline \multicolumn{8}{|l|}{ Carrera } \\
\hline Tecnología Médica & 22 & 62,86 & 13 & 37,14 & 1,83 & 1,28 & 2,63 \\
\hline Enfermería & 37 & 30,83 & 83 & 69,17 & 0,75 & 0,55 & 1,02 \\
\hline Medicina & 65 & 36,52 & 113 & 63,48 & 0,95 & 0,72 & 1,26 \\
\hline
\end{tabular}

Según los datos expuestos en la Tabla 3, los estudiantes con uso excesivo de TICs tienen una probabilidad de casi del doble de presentar síntomas de ansiedad (RR 1,9), en relación con los que no usan en exceso las TICs y esta diferencia es significativa (IC 95\% 1,46-2,47). Al analizar los síntomas depresivos encontramos que los alumnos con uso excesivo de TICs muestran más del doble de probabilidad de presentar este problema (RR 2,59) que aquellos sin uso excesivo de TICs, con una diferencia estadística significativa (IC95\% 1,66-4,06). 
Tabla 3. Uso excesivo de TICs y trastornos del estado del ánimo.

\begin{tabular}{|c|c|c|c|c|c|c|c|}
\hline \multirow{2}{*}{ Trastorno } & \multicolumn{2}{|c|}{ Con UE-TICs } & \multicolumn{2}{|c|}{ Sin UE-TICs } & \multirow{2}{*}{ RR } & \multirow{2}{*}{\multicolumn{2}{|c|}{ IC 95\% }} \\
\hline & $\mathrm{N}$ & $\%$ & $\mathrm{~N}$ & $\%$ & & & \\
\hline \multicolumn{8}{|l|}{ Ansiedad } \\
\hline Presente & 70 & 56,45 & 62 & 29,67 & 1,90 & 1,46 & 2,47 \\
\hline Ausente & 54 & 43,55 & 147 & 70,33 & & & \\
\hline \multicolumn{8}{|l|}{ Depresión } \\
\hline Presente & 37 & 29,84 & 24 & 11,48 & 2,59 & 1,66 & 4,06 \\
\hline Ausente & 87 & 70,16 & 185 & 88,52 & & & \\
\hline
\end{tabular}

\section{DISCUSIÓN}

Se trata de un estudio de tipo transversal realizado en una muestra aleatoria representativa de estudiantes de la Facultad de Ciencias Médicas de la Universidad de Cuenca, basado en una entrevista estructurada que fue validada localmente, por lo que puede haber limitaciones en la comparabilidad de los resultados, pues aunque en la mayoría de las investigaciones revisadas se menciona que las entrevistas se realizaron con base en los criterios de dependencia a drogas, es probable que las preguntas no hayan sido las mismas. Por otro lado, en razón de que en varios estudios asumen el concepto de adicción a las TICs (Echeburúa, 1999; Navarro-Marcilla y Rueda-Jaimes, 2007; Castañeda, 2010; Cruzado y col., 2006; Lam y col., 2011; Berner y Santander, 2012), mientras que en otros se sostiene que dicho término es aplicable únicamente a drogas y no al uso de las TICs (Yang, 2001; APA, 2002; Morrison y Gore, 2010; Domínguez y col., 2012; Fernández, 2013), se dificulta aún más la confrontación de los resultados. Además, debemos tener en cuenta que las características de las poblaciones fueron diversas en los distintos estudios, unas veces niños, otras estudiantes, adolescentes, adultos y hasta pacientes hospitalizados, en este estudio el $95 \%$ fueron estudiantes universitarios mayores de 19 años, con una edad promedio de 22 años.

Ya sea que el problema relacionado con la utilización de las TICs haya sido considerado como adicción o como uso excesivo, los resultados de las investigaciones revelaron valores variables en los diferentes países. Así, en Noruega la prevalencia de adicción al Internet fue de 1,98\%, en 3.237 adolescentes (Griffiths, 1998); en Gran Bretaña 18,3\%, en 371 estudiantes (Niemz y Griffiths, 2005); en Australia 9,6\%, en 293 jóvenes universitarios (Wang, 2001); en Estados Unidos 8,1\%, entre 283 estudiantes (Nichols y Nicki, 2004); en Corea del Sur 1,6\%, en 1.573 estudiantes de secundaria (Morahan-Martin y Schumacher, 2000). En España, en una muestra de 1.509 estudiantes menores encontraron $10,4 \%$ de adicción a la televisión, $10,2 \%$ al Internet, $12 \%$ al teléfono móvil, $12 \%$ a los videojuegos o juegos en el ordenador (Cabasés y col., 2011). En nuestro estudio el 37,2\% de los estudiantes presenta un uso exagerado de las TICs.

$\mathrm{Al}$ analizar los trastornos del estado de ánimo en quienes usan excesivamente las TICs, en este estudio encontramos que el 56,45\% presentaron síntomas de ansiedad, cuatro veces más que los hallazgos de Viñas y col. (2002) que fue de 13,3\%, y seis veces más que lo encontrado por Yang (2001) que fue $8,7 \%$. En nuestro estudio hallamos $29,84 \%$ casos de síntomas depresivos en estudiantes que usan excesivamente las TICs, frente al 22,35\% encontrado por Morrison y Gore (2010).

De acuerdo con el diseño de este estudio no podemos afirmar que el uso excesivo de las TICs constituye causa o consecuencia de los trastornos del estado de ánimo, simplemente hemos podido constatar que la probabilidad de síntomas depresivos y de ansiedad está incrementada de manera significativa en quienes usan excesivamente las TICs. Incluso existen algunas investigaciones en las que se ha encontrado que el uso de Internet puede servir para disminuir la depresión y la soledad más que para aumentarla (La Rose y col., 2001). 


\section{CONCLUSIONES}

En base a los resultados antes expuestos concluimos que el porcentaje de uso excesivo de TICs entre estudiantes de la Facultad de Ciencias Médicas de la Universidad de Cuenca es elevado. Las variables asociadas de manera significativa son la edad menor de 19 años, el sexo masculino y la carrera de Tecnología Médica. No está asociado de manera significativa al estado civil. Los alumnos que hacen uso excesivo de TICs tienen una mayor probabilidad estadísticamente significativa de presentar síntomas depresivos y de ansiedad.

\section{AGRADECIMIENTOS}

Los autores de este trabajo expresamos nuestra especial gratitud a todos quienes contribuyeron de distinta manera para la realización de la investigación y en forma especial a CEDIA por habernos financiado el proyecto, del mismo modo a las autoridades, docentes y estudiantes de las Facultades de Ciencias Médicas y de Odontología de la Universidad de Cuenca, quienes nos brindaron todas las facilidades y consiguieron que esta experiencia académica sea muy gratificante para todos los involucrados.

\section{BIBLIOGRAFÍA}

Akin, A., M. İskender, 2011. Internet addiction and depression, anxiety and stress. IOJES, 3(1), 138148.

American Psychiatric Association (APA), 2002. Manual diagnóstico y estadístico de los trastornos mentales DSM-IV-TR. En: Lopez-Ibor Alino J.J. (Ed.), Edición Español. Editorial Masson, Barcelona, España, 1088 pp.

Berner, J.E., J. Santander, 2012. Abuso y dependencia de internet: la epidemia y su controversia. Rev. Chil. Neuro-psiquiatr., 50(3), 181-190.

Blanco, M., M. Vigo, J. Fernández, M.M. Arbonès, 2002. Problemas conductuales con el uso del Internet. Anales Psicología, 18(2), 273-292.

Cabasés, J., J. Quintero, J. Correas, M.J. Pérez, T. Echaniz, 2011. Patrones de uso, abuso y dependencia a las tecnologías de la informacion en menores. Informe final. CON F.I.A.S y Consejo Escolar, Madrid, España. 323 págs. Descargado de http://www.madrid.org/cs/ Satellite $? c=C M \_$Actuaciones_FA\&cid=1142433011852\&idConsejeria $=1109266187254 \&$ idList Consj $=1109265444710 \&$ language $=$ es \&pagename $=$ ComunidadMadrid\% 2 FEstructura $\&$ sm $=110$ 9265843983 en febrero 2014.

Castañeda, E., 2010. Adicción al trabajo (workaholism). Patología psicosocial del siglo XXI. Salud trab. (Maracay), 18(1), 57-66.

Cruzado, L., L. Matos, R. Kendall, 2006. Adicción a internet: Perfil clínico y epidemiológico de pacientes hospitalizados en un instituto nacional de salud mental. Rev. Méd. Hered., 17(4), 196205.

Diccionario de la Real Academia Española (DRAE) (22 ${ }^{\mathrm{a}}$ Edición), 2001. Descargado de http://www.rae.es/recursos/diccionarios/drae\#sthash.2AnBE1bj.dpuf el 28 de abril 2014.

Domínguez, C., S. Geijo, I. Sánchez, C. Imaz, G. Cabús, 2012. Revisión de los mecanismos implicados en el uso problemático de Internet. Rev. Asoc. Esp. Neuropsiq., 32(116), 691-705.

Echeburúa, E., 1999. Adicciones sin drogas? Las nuevas adicciones: juego, sexo, comida, compras, trabajo, internet (2 Edición). Colección PREGUNTAS, 144 pp.

Fernández, N., 2013. Trastornos de conducta y redes sociales en Internet. Salud Mental, 36, 521-527. 
Griffiths, M., 1998. Internet addiction: Does it really exist? In: Gackenbach, J. (Ed.) Psychology and the Internet: Intrapersonal, interpersonal and transpersonal implications.Psychology and the Internet. Academic Press, San Diego, CA, USA, 61-75.

Hamilton, M., 1960. A rating scale for depression. J. Neurol. Neurosurg. Psychiatry, 23, 56-62.

Kraut, R., M. Patterson, V. Lundmark, S. Kiesler, T. Mukopadhyay, W. Scherlis, 1998. Internet paradox. A social technology that reduces social involvement and psychological well-being? Am. Psychol., 53(9), 1017-31.

Kyunghee, K., R. Eunjung, C. Mi-Young, Y. Eun-Ja, C. So-Young, S. Jeong-Seok, N. Bum-Woo, 2006. Internet addiction in Korean adolescents and its relation to depression and suicidal ideation: A questionnaire survey. Int. J. Nurs. Stud., 43, 185-192.

Lam, N., H. Contreras, E. Mori, M. Nizama, C. Gutiérrez, W. Hinostroza, E. Torrejón, R. Hinostroza, E. Coaquira, W.D. Hinostroza, 2011. Adicción a internet: desarrollo y validación de un instrumento en escolares adolescentes de Lima, Perú. Rev. Peru Med. Exp. Salud Pública, 28(3), 462-9.

La Rose, R., M.S. Eastin, J. Gregg, 2001. Reformulating the Internet paradox: Social cognitive explanations of Internet use and depression. J. Online Behav., 1(2). Descargado de http://www.behavior.net/JOB/v1n1/paradox.html el 29 de abril 2014.

Morahan-Martin, J., P. Schumacher, 2000. Incidence and correlates of pathological Internet use among college students. Comput. Hum. Behav., 16, 13-29.

Morrison, C.M., H. Gore, 2010. The relationship between excessive Internet use and depression: a questionnaire-based study of 1,319 young people and adults. Psychopathology, 43(2), 121-6.

Navarro-Mancilla, A., G. Rueda-Jaimes, 2007. Adicción a Internet: revisión crítica de la literatura. Rev. Colomb. Psiquiatr., 36(4), 691-700.

Nichols, L.A., R. Nicki, 2004. Development of a psychometrically sound Internet addiction scale: a preliminary step. Psychol. Addict. Behav., 18(4), 381-4.

Niemz, K., M. Griffiths, P. Banyard, 2005. Prevalence of pathological Internet use among university students and correlations with self-esteem, the General Health Questionnaire (GHQ), and disinhibition. CyberPsychol. Behav., 8(6), 562-70.

Organización Mundial de la Salud (OMS), 2012. La depresión. Nota descriptiva №369. Descargado de http://www.who.int/mediacentrelfactsheets/fs369/es/ el 29 de abril 2014.

Viñas, F., J.J. Ferrer, E. Villar, B. Caparrós, I. Pérez, M. Cornellá, 2002. Internet y Psicopatología: las nuevas formas de comunicación y su relación con diferentes índices de psicopatología. Clinica y Salud, 13(3), 235-256.

Wang, W., 2001. Internet dependency and psychosocial maturity among college students. Int. J. Hum. Comput. Stud., 55(6), 919-38.

Wingo, P.A., 1996. Epidemiología aplicada a la salud reproductiva. Organización Mundial de la Salud (OMS), $478 \mathrm{pp}$

Yang, C.K., 2001. Sociopsychiatric characteristics of adolescents who use computers to excess. Acta Psychiatr. Scand., 104(3), 217-222.

Young, K.S., R.C. Rogers, 1998. The relationship between depression and Internet addiction. CyberPsychol. Behav., 1(1), 25-28. 